\title{
An Improved Antenna Group Delay Measurement Method Using a Three-antenna Extrapolation Technique
}

\author{
Haoyu LIN ${ }^{1,2}$, Zhenfei SONG ${ }^{2}$, Xuetian $W A N G^{1}$, Hongmin GAO \\ ${ }^{1}$ Dept. of Information and Electronics, Beijing Inst. of Technology, ZhongGuanCunNanDaJie 5, 100081, Beijing, China \\ ${ }^{2}$ National Inst. of Metrology, BeiSanHuanDongLu 18, 100029, Beijing, China
}

\{linhy, songzf\}@nim.ac.cn, \{wangxuetian,gaohm\}@bit.edu.cn

Submitted April 15, 2017 / Accepted June 24, 2017

\begin{abstract}
In order to minimize the error due to multiple reflections between antennas in the conventional group delay (GD) measurement, an improved antenna GD measurement method is proposed. In this method, antenna group delay is measured as a function of distances using a three-antenna extrapolation method. The GD is determined by averaging a set of measured GD values according to a derived multiple-reflection error model. Measurement in frequency band of (1575.42 \pm 16$) \mathrm{MHz}$ for a circularly polarized helical antenna is presented, which gives the detail measurement procedures and validates the method. The uncertainty evaluation for this measurement was carried out as well, and an expanded uncertainty of $0.20 \mathrm{~ns}(k=2)$ has been achieved. One more measurement example in frequency band of $(4000 \pm 10) \mathrm{MHz}$ for a standard gain horn antenna with an expanded uncertainty of $0.12 \mathrm{~ns}(k=2)$ is also presented briefly in this paper.
\end{abstract}

\section{Keywords}

Group delay, antenna measurement, three-antenna extrapolation, uncertainty evaluation

\section{Introduction}

Antenna is an essential part of a transmitting or receiving system that is designed to radiate or to receive electromagnetic waves [1]. Antenna performance is characterized by various parameters which can be obtained through measurements [2], [3]. In the past, antenna measurements always aimed at gain, pattern, efficiency and some other main parameters [4-10]. With the development of satellite navigation, remote sensing, modern communication, et al., antenna group delay (GD) is paid more and more attentions nowadays. Especially for global navigation satellite systems (GNSS), antenna GD measurement accuracy will set the boundaries of how well it will perform [11-15]. The group delay of the antenna versus aspect angle is defined in the standard of Radio Technical Commission for Aeronautics (RTCA), and its limit is also specified from $0.65 \mathrm{~ns}$ to $2.5 \mathrm{~ns}$ related to the elevation angle at GPS L1 frequency $(1575.42 \mathrm{MHz})$ which shows the requirements for high accuracy antenna GD measurement [16].

Standard antenna method, two identical antenna method and three-antenna method are three conventional antenna GD measurement methods, and the three-antenna method is the most accurate one among them [17], [18]. All these methods work at some fixed distances and measurement error caused by multiple reflections between transmitting (Tx) and receiving ( $\mathrm{Rx}$ ) antennas is hardly taken into consideration. In this paper, an improved antenna GD measurement method based on three-antenna extrapolation technique is proposed. In this method, no standard antenna is required and the measurement error caused by multiple reflections can be reduced effectively.

In this paper, the measurement methodology is described in Sec. 2, and Section 3 gives the measurement procedures and results. The uncertainty evaluation is discussed in Sec. 4, and the conclusion is given in Sec. 5.

\section{Methodology}

The group delay is defined as follows [19]:

$$
G D(f)=-\frac{1}{360} \frac{\mathrm{d} \varphi(f)}{\mathrm{d} f}
$$

where $G D(f)$ is group delay (in second), $f$ is frequency, and $\varphi(f)$ is phase function (in degree). For engineering purpose, group delay is often calculated using the following approximation:

$$
G D\left(f_{\mathrm{c}}\right)=-\frac{1}{360} \frac{\varphi\left(f_{2}\right)-\varphi\left(f_{1}\right)}{f_{2}-f_{1}}
$$

where $f_{\mathrm{c}}$ is center frequency, $f_{1}$ and $f_{2}$ are any two different frequencies on opposite sides of $f_{\mathrm{c}}$ within the required frequency range. 


\subsection{Conventional Three-antenna GD Measurement}

Three-antenna method does not need any standard antenna, but two auxiliary antennas (marked: A and B) are required besides the antenna under test (AUT, marked: C). The measurement is divided into three groups and two antennas are contained in each group. The measuring sequence is shown in Tab. 1.

Taking Group 1 for example, equation (3) is obtained:

$$
\varphi_{\mathrm{AB}}=\varphi_{\mathrm{ab}}-\varphi_{\mathrm{d}}+\varphi_{0}
$$

where $\varphi_{\mathrm{AB}}$ is the total phase function of the insertion loss measured between Tx and $\mathrm{Rx}$ antennas, $\varphi_{\mathrm{ab}}$ is the sum of the phase function of antenna $\mathrm{A}$ and $\mathrm{B}, \varphi_{\mathrm{d}}$ is the phase function associated with measuring distance, $\varphi_{0}$ is the phase function of the measurement system itself measured in case of a through connection, which is defined as the Tx output and Rx input transmission lines connected directly.

Taking (3) into (2), the group delay can be obtained, as shown in (4):

$$
G D_{\mathrm{AB}}=G D_{\mathrm{ab}}-G D_{\mathrm{d}}+G D_{0}
$$

where

$$
\begin{gathered}
G D_{\mathrm{ab}}=G D_{\mathrm{a}}+G D_{\mathrm{b}}, \\
G D_{\mathrm{d}}=-\frac{d}{c} .
\end{gathered}
$$

$G D_{\mathrm{AB}}, G D_{\mathrm{ab}}, G D_{\mathrm{d}}$ and $G D_{0}$ are group delays corresponding to phase functions $\varphi_{\mathrm{AB}}, \varphi_{\mathrm{ab}}, \varphi_{\mathrm{d}}$ and $\varphi_{0}, G D_{\mathrm{a}}$ and $G D_{\mathrm{b}}$ are group delays of antenna $\mathrm{A}$ and $\mathrm{B}$ respectively, $d$ is the distance between the apertures of Tx and Rx antennas, and $c$ is the speed of light.

After Group 1 is finished, $G D_{\mathrm{ab}}$ is calculated by solving the above equations. Similarly, the $G D_{\mathrm{ac}}$ and $G D_{\mathrm{bc}}$ are calculated after Group 2 and 3 are finished. Finally $G D_{\mathrm{c}}$ is calculated by solving (7):

$$
\left\{\begin{array}{l}
G D_{\mathrm{a}}+G D_{\mathrm{b}}=G D_{\mathrm{ab}}, \\
G D_{\mathrm{a}}+G D_{\mathrm{c}}=G D_{\mathrm{ac}}, \\
G D_{\mathrm{b}}+G D_{\mathrm{c}}=G D_{\mathrm{bc}}
\end{array}\right.
$$

\subsection{Improved GD Measurement Method}

During the process of antenna measurement, the electromagnetic wave propagates from $\mathrm{Tx}$ antenna to $\mathrm{Rx}$ antenna, but only a part of the arriving wave is received by $\mathrm{Rx}$ antenna and other is reflected back into the space. After the reflected wave arriving at Tx antenna, a part of it is reflected back towards Rx antenna similarly, so in this way the multiple reflections are formed between the two antennas and this process is repeated endlessly. Due to multiple reflections, $\varphi(f)$ and $\mathrm{d} \varphi(f)$ are measured differently at different distances, and this causes group delay measurement errors obviously.

\begin{tabular}{|c|c|c|}
\hline & Tx antenna & Rx antenna \\
\hline Group 1 & A & B \\
\hline Group 2 & A & C \\
\hline Group 3 & B & C \\
\hline
\end{tabular}

Tab. 1. Measuring sequence.

Here, in order to simplify the analysis process, we ignore higher order reflections and only consider that the wave is reflected by Rx and Tx antennas for one time individually. The reflected wave is superposed with direct wave and then they are received together by $\mathrm{Rx}$ antenna.

The expression of the electric field at the aperture of $\mathrm{Rx}$ antenna is:

$$
\begin{aligned}
E & =\frac{E_{0}}{d} \exp (-\mathrm{j} k d) \\
& +\frac{E_{0}}{d} \exp (-\mathrm{j} k d) \beta_{1} \frac{1}{d} \exp (-\mathrm{j} k d) \beta_{2} \frac{1}{d} \exp (-\mathrm{j} k d) \\
& =\frac{E_{0}}{d} \exp (-\mathrm{j} k d)\left(1+\beta \frac{1}{d^{2}} \exp (-2 \mathrm{j} k d)\right)
\end{aligned}
$$

where $E$ represents electric field, $E_{0}$ is the amplitude of $E$, $\beta_{1}$ and $\beta_{2}$ are scattering characteristics of $\mathrm{Rx}$ and $\mathrm{Tx}$ antennas looking into the antenna aperture, $\beta=\beta_{1} \beta_{2}$, $k=2 \pi / \lambda[20]$.

Using Euler's formula, equation (8) can be written as:

$$
E=\frac{E_{0}}{d} \exp (-\mathrm{j} k d)\left[1+\beta \frac{1}{d^{2}} \cos (2 k d)-\mathrm{j} \beta \frac{1}{d^{2}} \sin (2 k d)\right] \text {. }
$$

In most of our practical measurements, for minimizing the antenna multiple reflections, the measurement is carried out as far as possible subject to a sufficient signalnoise ratio, which results that $\beta / d^{2}$ is much smaller than one, and (10) can be assumed:

$$
1+\beta \frac{1}{d^{2}} \cos (2 k d) \approx 1
$$

So equation (9) can be written as:

$$
\begin{aligned}
E & =\frac{E_{0}}{d} \exp (-\mathrm{j} k d)\left[1-\mathrm{j} \beta \frac{1}{d^{2}} \sin (2 k d)\right] \\
= & \frac{E_{0}}{d} \sqrt{1+\left[\beta \frac{1}{d^{2}} \sin (2 k d)\right]^{2}} . \\
& \exp \left[-\mathrm{j} k d-\mathrm{j} \operatorname{atan}\left(\beta \frac{1}{d^{2}} \sin (2 k d)\right)\right] .
\end{aligned}
$$

Also because $\beta / d^{2}$ is much smaller than one, the following approximation is made:

$$
\operatorname{atan}\left[\beta \frac{1}{d^{2}} \sin (2 k d)\right] \approx \beta \frac{1}{d^{2}} \sin (2 k d) .
$$

So equation (11) can be written as:

$E=\frac{E_{0}}{d} \sqrt{1+\left[\beta \frac{1}{d^{2}} \sin (2 k d)\right]^{2}} \exp \left\{-\mathrm{j}\left[k d+\beta \frac{1}{d^{2}} \sin (2 k d)\right]\right\}$. 
Clearly, the phase of $E$ is:

$$
\varphi_{\mathrm{m}}=k d+\beta \frac{1}{d^{2}} \sin (2 k d) .
$$

The phase error caused by multiple reflections is:

$$
\Delta \varphi=\beta \frac{1}{d^{2}} \sin (2 k d)=\beta \frac{1}{d^{2}} \sin \left(\frac{4 \pi f d}{c}\right) .
$$

So the group delay multiple-reflection error model is:

$$
\Delta G D=-\frac{1}{2 \pi} \frac{\mathrm{d} \Delta \varphi}{\mathrm{d} f}=-\frac{2 \beta}{c d} \cos \left(\frac{4 \pi f}{c} d\right)=-\frac{2 \beta}{c d} \cos (2 k d)
$$

where $\Delta G D$ is group delay measurement error caused by multiple reflections.

Using (16) to calculate $\Delta G D$ and correct the measurement results is a direct way to eliminate $\Delta G D$. In order to calculate $\Delta G D$ accurately, $\beta_{1}$ and $\beta_{2}$ should be determined in prior, and this process is very complicated and timeconsuming.

In the improved method, firstly $G D_{\mathrm{c} n}$ is measured using extrapolation range where $G D_{\mathrm{c} n}(n=1,2,3, \ldots, N)$ is the group delay of antenna $\mathrm{C}$ measured at $N$ different distances, and then the mean value of $G D_{\mathrm{c} n}$ is calculated as the measurement result as shown in (17). According to (16), $\Delta G D$ is a cosine function of $d$ and the period is $\lambda / 2$, so its positive parts and negative parts are counteracted by averaging, and $\triangle G D$ is reduced in this way.

$$
G D_{\text {cMean }}=\frac{G D_{\mathrm{c} 1}+G D_{\mathrm{c} 2}+G D_{\mathrm{c} 3}+\cdots+G D_{\mathrm{cN}}}{N}
$$

\section{Measurement Example}

\subsection{Antenna Extrapolation Range}

The measurement is performed in a newly constructed antenna extrapolation range at the National Institute of Metrology (NIM), China [2].The transmitting carriage of the system can move freely along two 10-meter-long precision rails in direction of the longitudinal axis of the range (z-axis). The rails are aligned and installed precisely on the chamber ground with maximum deviations of $0.08 \mathrm{~mm}$ in vertical and $0.06 \mathrm{~mm}$ in horizontal, respectively. The transmitting carriage is guided by a ball-screw driver with a maximum speed of $80 \mathrm{~mm} / \mathrm{s}$ and positioning resolution of $1 \mu \mathrm{m}$. The actual relative movement of the carriage is determined by a laser interferometer with error of $0.1 \mu \mathrm{m}$. The photo of the chamber is shown in Fig. 1.

A vector network analyzer (VNA) together with external mixers are used in the radio frequency (RF) measurement subsystem of the antenna extrapolation range. The VNA provides local oscillation (LO) signal and receives intermediate frequency (IF) signal, which is the product of frequency mixing. LO signal goes into reference/test mixers individually after processed by $\mathrm{LO} / \mathrm{IF}$ distribution

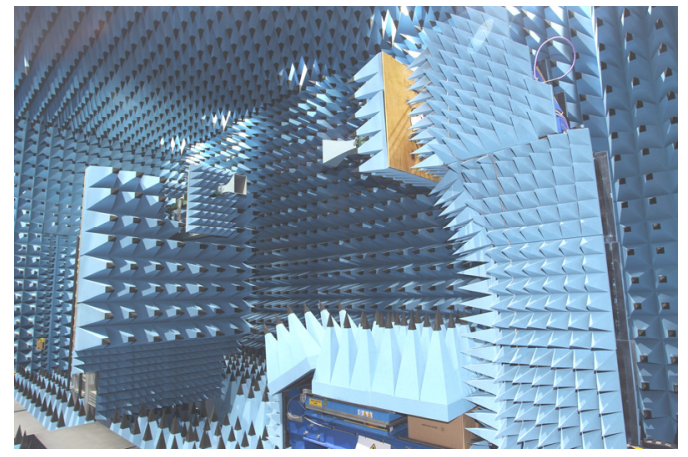

Fig. 1. Antenna extrapolation range.

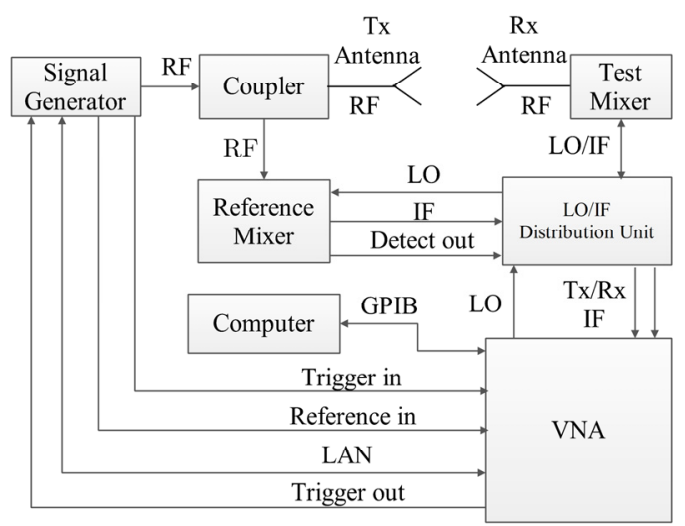

Fig. 2. System schematic of antenna extrapolation range

unit, and then mixed with RF transmitting and receiving signal to generate IF signal. System schematic is illustrated in Fig. 2.

\subsection{Measurement Procedures}

In this part, practical measurement of group delay for a circularly polarized helical antenna is given. The main measurement setup involved is summarized in Tab. 2. The measurement starts at 5 meters and with an extrapolation range of $0.96 \mathrm{~m}$, i.e., five times wavelength with respect to $1575.42 \mathrm{MHz}\left(5 \lambda_{\mathrm{L} 1}\right)$.

The system is set up according to Fig. 2. Cables with $\mathrm{N}$ type connectors are used to connect antennas and other equipment in the system. Taking Group 1 as an example, firstly antenna B is mounted on Rx tower, and then it is aligned using optical alignment system until the normal of the antenna aperture is parallel with z-axis. Then, antenna A is mounted on transmitting carriage and its attitude is adjusted using the adjustable mounts until the apertures of $\mathrm{A}$ and $\mathrm{B}$ are well aligned.

\begin{tabular}{|l|l|}
\hline \multicolumn{1}{|c|}{ Parameters } & \multicolumn{1}{c|}{ Values } \\
\hline Frequency band & $(1575.42 \pm 16) \mathrm{MHz}$ \\
\hline Frequency step & $0.5 \mathrm{MHz}$ \\
\hline Auxiliary antenna type & $\begin{array}{l}\text { Linearly polarized standard gain } \\
\text { horn (SGH) antenna }\end{array}$ \\
\hline AUT type & Circularly polarized helical antenna \\
\hline Extrapolation range & $(5 \sim 5.96) \mathrm{m}$ \\
\hline Position points $(\mathrm{N})$ & 80 \\
\hline
\end{tabular}

Tab. 2. Measurement parameters. 
First of all, a so-called "through connection" measurement is performed. Tx and $\mathrm{Rx}$ antennas are taken off and the cables are connected together using an adaptor. The signal generator output power is adjusted to a proper level to ensure the mixers working in a linear region, and then $G D_{0}$ is measured. The GD of the adaptor itself should be measured individually to compensate $G D_{0}$. Throughconnection is performed before each group and after the last group, four times in total.

Then Antenna A and B are mounted on transmitting carriage and $\mathrm{Rx}$ towers respectively and aligned once again, and then $G D_{\mathrm{AB}}$ is obtained at different distances by the insertion loss measurement. Following a similar procedure, the $G D_{\mathrm{AC}}$ and $G D_{\mathrm{BC}}$ are determined.

The group delay of AUT on each position $G D_{\mathrm{c} n}$ is calculated according to theory described in Sec. 2.2, and then $G D_{\text {cMean }}$ is calculated according to (17).

The mismatch between the antenna and the cable it connects can lead to GD measuring error, so mismatch correction is necessary. Considering mismatch, the following equations are obtained from (7):

$$
\left\{\begin{array}{l}
G D_{\mathrm{a}}+\Delta G D_{\mathrm{aT}}+G D_{\mathrm{b}}+\Delta G D_{\mathrm{bR}}=G D_{\text {abMis }} \\
G D_{\mathrm{a}}+\Delta G D_{\mathrm{aT}}+G D_{\mathrm{c}}+\Delta G D_{\mathrm{cR}}=G D_{\text {acMis }} \\
G D_{\mathrm{b}}+\Delta G D_{\mathrm{bT}}+G D_{\mathrm{c}}+\Delta G D_{\mathrm{cR}}=G D_{\mathrm{bcMis}}
\end{array}\right.
$$

where $\Delta G D_{i j}$ is GD measurement error caused by the mismatch between antenna $i(i=\mathrm{a}, \mathrm{b}$ or $\mathrm{c})$ and the cable it connects, and $j(\mathrm{~T}$ or $\mathrm{R}$ ) is the notation of transmitting or receiving cable. $G D_{k I \text { Mis }}$ is the sum of group delay of antenna $k$ and $l(k=\mathrm{a}$ or $\mathrm{b}, l=\mathrm{b}$ or $\mathrm{c})$ considering mismatch.

By solving (18), the group delay of antenna $\mathrm{C}\left(G D_{\mathrm{c}}\right)$ after mismatch correction can be obtained:

$$
\begin{aligned}
G D_{\mathrm{c}}= & \frac{\left(G D_{\mathrm{bcMis}}+G D_{\mathrm{acMis}}-G D_{\mathrm{abMis}}\right)}{2} \\
& +\frac{\Delta G D_{\mathrm{bR}}-\Delta G D_{\mathrm{bT}}}{2}-\Delta G D_{\mathrm{cR}} .
\end{aligned}
$$

Comparing $G D_{\mathrm{c}}$ with the solution of (7), the mismatch correction factor is obtained as shown in (20):

$$
\begin{aligned}
\Delta G D_{\mathrm{cMiscor}} & =\frac{\Delta G D_{\mathrm{bR}}-\Delta G D_{\mathrm{bT}}}{2}-\Delta G D_{\mathrm{cR}}, \\
\Delta G D_{\mathrm{bR}}\left(\frac{f_{1}+f_{2}}{2}\right) & =\Delta G D_{\mathrm{cR}}\left(\frac{f_{1}+f_{2}}{2}\right) \\
& =-\frac{1}{360} \frac{\Delta \varphi_{\mathrm{R}}\left(f_{2}\right)-\Delta \varphi_{\mathrm{R}}\left(f_{1}\right)}{f_{2}-f_{1}}, \\
\Delta G D_{\mathrm{bT}}\left(\frac{f_{1}+f_{2}}{2}\right) & =-\frac{1}{360} \frac{\Delta \varphi_{\mathrm{bT}}\left(f_{2}\right)-\Delta \varphi_{\mathrm{bT}}\left(f_{1}\right)}{f_{2}-f_{1}}, \\
\Delta \varphi_{\mathrm{R}} & =\angle\left(1+\Gamma_{\mathrm{R}}\right), \\
\Delta \varphi_{\mathrm{bT}} & =\angle\left(1+\Gamma_{\mathrm{B}}\right)
\end{aligned}
$$

where $\Delta \varphi_{R}$ is the phase measuring error caused by the mismatch between Rx antenna (i.e., antenna $\mathrm{B}$ and $\mathrm{C}$ specified in Tab. 1) and the cable it connects, $\Delta \varphi_{\mathrm{bT}}$ is the phase measuring error caused by the mismatch between antenna $\mathrm{B}$ and its cable when B is used as a transmitting antenna. $\Gamma_{\mathrm{R}}$ and $\Gamma_{\mathrm{B}}$ are the reflection coefficients looking into the Rx cable and antenna B feeding port respectively [19].

The final group delay measurement result of antenna $\mathrm{C}$ after mismatch correction is shown in (25):

$$
G D_{\mathrm{c}}=G D_{\mathrm{cMean}}+\Delta G D_{\mathrm{cMisCor}} \text {. }
$$

\subsection{Measurement Results}

The $G D_{\mathrm{c} N}$ values at $1575.42 \mathrm{MHz}$ frequency with respect to different distances are shown in Fig. 3. A curve fitted according to (16) and $G D_{\mathrm{cN}}$ is also shown in this figure and its expression is $C_{\text {Fitted }}=\left(A_{1} / d\right) \cos (2 k d)+A_{2}$, where $A_{1}$ and $A_{2}$ are frequency-dependent fitting coefficients. We can see that $G D_{\mathrm{c} N}$ varies with distance periodically and the period is approximately $\lambda_{\mathrm{L} 1} / 2$, the same as the fitted curve in accordance with (16). Although the amplitudes of $G D_{\mathrm{c} N}$ at some distances are a little different from the fitted curve, this is still reasonable considering the cable flexure, the random error and other reflections from the absorbers on the walls, ceiling and floor. Generally speaking, the variation of $G D_{\mathrm{cN}}$ is in accordance with (16) mainly caused by multiple reflections and it is proved that $\Delta G D$ can be reduced according to the theory described in Sec. 2.2. In fact, the measurement process of three-antenna extrapolation method is similar as that of conventional three-antenna method when it is performed at $N$ different

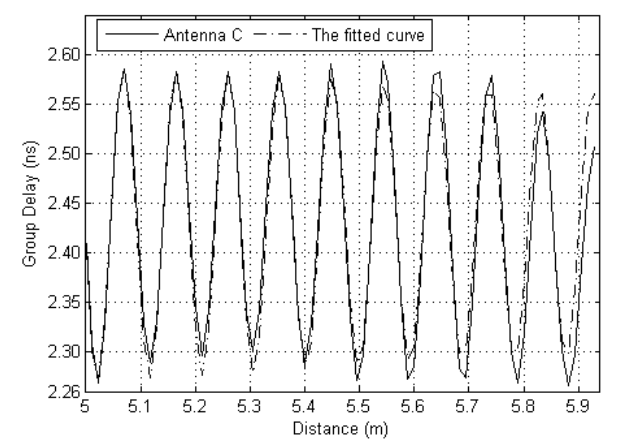

Fig. 3. The $G D_{\mathrm{c} N}$ values and the fitted curve at GPS L1 frequency with respect to different distances.

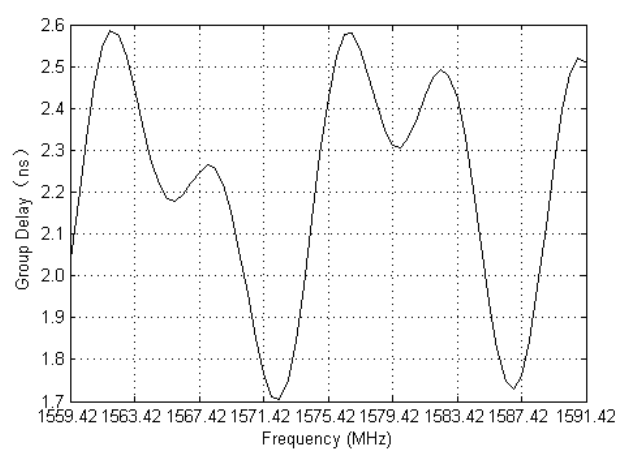

Fig. 4. $G D_{\mathrm{c}}$ at different frequencies. 
distances in series. So according to Fig. 3, the measurement results vary from $2.27 \mathrm{~ns}$ to $2.59 \mathrm{~ns}$ if using conventional three-antenna method compared with that of $2.43 \mathrm{~ns}$ if using the proposed method, and it is proved that the proposed method is improved in measurement accuracy.

$G D_{\text {c }}$ at different frequencies is shown in Fig. 4, which shows the GD variation with frequency of antenna $C$.

\subsection{Measurement for a Standard Gain Horn Antenna}

One more measurement example is performed in frequency band of $(4000 \pm 10) \mathrm{MHz}$ with frequency step of $0.5 \mathrm{MHz}$ using Narda $643 \mathrm{SGH}$ antenna following the same procedure with the above measurement. In this measurement, antennas are connected to waveguides directly without using waveguide-to-coaxial transducer in order to guarantee better stability of the system.

The antenna GD measurement result at $4 \mathrm{GHz}$ frequency point vary from $1.38 \mathrm{~ns}$ to $1.45 \mathrm{~ns}$ with distance, and the mean value is $1.42 \mathrm{~ns}$. In the frequency band of $(4000 \pm 10) \mathrm{MHz}$, the measurement results vary from $0.86 \mathrm{~ns}$ to $1.78 \mathrm{~ns}$ with frequency and an expanded uncertainty of $0.12 \mathrm{~ns}$ is achieved.

\section{Uncertainty Evaluation}

The uncertainty budget for the measurement in GPS L1 frequency band is shown in Tab. 3. The analysis according to [21] of each source will be given as follows:

- $u_{\mathrm{m}}$ : measurement repeatability:

Because group delay measurement result $G D_{\mathrm{c}}$ is the mean value of $N$ times measurement results, $u_{\mathrm{m}}$ is determined by (27):

$$
u_{\mathrm{m}}=\frac{s t d_{\mathrm{mMax}}}{\sqrt{N}}=\frac{0.1604}{\sqrt{80}} \mathrm{~ns}=0.0179 \mathrm{~ns}
$$

where $s t d_{\mathrm{mMax}}$ is the maximum standard deviation of $N$ times measurement results over all the frequency points.

- $u_{\mathrm{AC}}$ : antenna connection repeatability:

To evaluate $u_{\mathrm{AC}}$, the antenna is connected and reconnected to the cable 10 times and GD is measured every time when the antenna is connected. $u_{\mathrm{AC}}$ is shown as follows:

$$
u_{\mathrm{AC}}=s t d_{\mathrm{ACMax}}=0.0473 \mathrm{~ns}
$$

where $s t d_{\mathrm{ACMax}}$ is the maximum standard deviation of the 10 times measurement results over all the frequency points.

- $u_{\mathrm{CC}}$ : cable connection repeatability:

The evaluation process of $u_{\mathrm{CC}}$ is similar with that of $u_{\mathrm{AC}}$ where $\mathrm{Tx}$ and $\mathrm{Rx}$ cables are connected and reconnected 10 times instead and the result is $0.0094 \mathrm{~ns}$.

- $u_{\mathrm{CF}}$ : cable flexure:

During the process of extrapolation measurement, the LO cable is flexing with the movement of the transmitting carriage, and as a result GD of the measurement system itself is changing.

To evaluate the $u_{\mathrm{CF}}$, a section of LO cable on transmitting carriage is bended at different parts and GD is measured under through-connection. $u_{\mathrm{CF}}$ is determined by (29):

$$
u_{\mathrm{CF}}=\frac{\Delta G D_{\mathrm{SMax}}}{\sqrt{2}}=\frac{0.0440}{\sqrt{2}} \mathrm{~ns}=0.0311 \mathrm{~ns}
$$

where $\Delta G D_{\mathrm{SMax}}$ is the maximum deviation among the group delay values measured when the cable is bended at different parts over all the frequency points.

- $u_{\mathrm{D}}$ : RF subsystem drift:

During the measuring process, GD of the measurement system itself is drifting with time, and as a result, $u_{\mathrm{D}}$ is caused. As described in Sec. 3.2, $G D_{0}$ is measured 4 times in total from $G D_{01}$ to $G D_{04}$, and equation (30) is obtained:

$$
G D_{0 i}-G D_{0 j}=\Delta G D_{0 i j}
$$

where $i=2,3,4, j=i-1, \Delta G D_{0 i j}$ is the deviation of $G D_{0 i}$ and $G D_{0 j} . u_{\mathrm{D}}$ is shown in (31):

$$
u_{\mathrm{D}}=\frac{\Delta G D_{0 \mathrm{Max}}}{\sqrt{3}}=\frac{0.0105}{\sqrt{3}} \mathrm{~ns}=0.0061 \mathrm{~ns}
$$

where $\Delta G D_{0 \text { Max }}$ is the maximum $\Delta G D_{0 i j}$ over all the frequency points.

- $u_{\mathrm{AM}}$ : antenna misalignment:

Azimuth, pitch and roll are three kinds of typical error angles in antenna misalignment, and the uncertainty caused by them are $u_{\mathrm{AM} 1}, u_{\mathrm{AM} 2}$ and $u_{\mathrm{AM} 3}$ respectively.

\begin{tabular}{|c|c|c|c|c|c|c|}
\hline NO. & Symbol & Source of Uncertainty & $\begin{array}{c}\text { Value } \\
(\mathbf{n s})\end{array}$ & $\begin{array}{c}\text { Probability } \\
\text { Distribution }\end{array}$ & Divisor & $\begin{array}{c}\text { Standard } \\
\text { Uncertainty (ns) }\end{array}$ \\
\hline 1 & $u_{\mathrm{m}}$ & Measurement Repeatability & 0.0179 & Normal & 1 & 0.0179 \\
\hline 2 & $u_{\mathrm{AC}}$ & Antenna Connection Repeatability & 0.0473 & Normal & 1 & 0.0473 \\
\hline 3 & $u_{\mathrm{CC}}$ & Cable Connection Repeatability & 0.0094 & Normal & 1 & 0.0094 \\
\hline 4 & $u_{\mathrm{CF}}$ & Cable Flexure & 0.0440 & U-Shaped & $2^{1 / 2}$ & 0.0311 \\
\hline 5 & $u_{\mathrm{D}}$ & Drift & 0.0105 & Rectangular & $3^{1 / 2}$ & 0.0061 \\
\hline 6 & $u_{\mathrm{AM}}$ & Antenna Misalignment & 0.1143 & U-Shaped & $2^{1 / 2}$ & 0.0808 \\
\hline 7 & $u_{\mathrm{SN}}$ & Systerm Nonlinearity & 0.0084 & Rectangular & $3^{1 / 2}$ & 0.0048 \\
\hline \hline$/$ & $U_{\mathrm{c}}$ & Combined Standard Uncertainty & $/$ & Normal & $/$ & 0.1010 \\
\hline$/$ & $U$ & Expanded Uncertainty $(k=2)$ & Normal & $/$ & 0.20 \\
\hline
\end{tabular}

Tab. 3. Uncertainty budget. 
According to our practices, azimuth error angle is often less than $0.5^{\circ}$, so when evaluating $u_{\mathrm{AM} 1}$, GD is measured under azimuth angle $\pm 0.5^{\circ}$ and compared with the one measured under $0^{\circ}$ individually. $u_{\mathrm{AM} 1}$ is shown in (32):

$$
u_{\mathrm{AM} 1}=\frac{\Delta G D_{\mathrm{AM} 1 \mathrm{Max}}}{\sqrt{2}}=\frac{0.0800}{\sqrt{2}} \mathrm{~ns}=0.0566 \mathrm{~ns}
$$

where $\Delta G D_{\mathrm{AM} 1 \mathrm{Max}}$ is the maximum absolute value of the above comparing results over all the frequency points.

The evaluation process of $u_{\mathrm{AM} 2}$ and $u_{\mathrm{AM} 3}$ is similar with that of $u_{\mathrm{AM} 1}$, where $u_{\mathrm{AM} 2}=0.0566 \mathrm{~ns}$ and $u_{\mathrm{AM} 3}=$ $0.0116 \mathrm{~ns}$.

Finally $u_{\mathrm{AM}}$ is calculated as shown in (33):

$$
\begin{aligned}
u_{\mathrm{AM}} & =\sqrt{u_{\mathrm{AM} 1}^{2}+u_{\mathrm{AM} 2}^{2}+u_{\mathrm{AM} 3}^{2}} \\
& =\sqrt{0.0566^{2}+0.0566^{2}+0.0116^{2}} \mathrm{~ns}=0.0808 \mathrm{~ns} .
\end{aligned}
$$

- $u_{\mathrm{SN}}$ : RF subsystem nonlinearity:

During the process of extrapolation measurement, the received signal strength varies with $d$. Because of the nonlinearity of the mixers, different input power will cause different responses, and as a result $u_{\mathrm{SN}}$ is caused. To evaluate $u_{\mathrm{SN}}$, the distance is set to $5.5 \mathrm{~m}$ and GD measured under signal generator output power $0 \mathrm{dBm},-10 \mathrm{dBm},-15 \mathrm{dBm}$ and $-20 \mathrm{dBm}$ are compared with the one measured under $-5 \mathrm{dBm}$ individually. $u_{\mathrm{SN}}$ is shown in (34):

$$
u_{\mathrm{SN}}=\frac{\Delta G D_{\mathrm{SNMax}}}{\sqrt{3}}=\frac{0.0084}{\sqrt{3}} \mathrm{~ns}=0.0048 \mathrm{~ns}
$$

where $\Delta G D_{\mathrm{SNMax}}$ is the maximum absolute value of the above comparing results over all the frequency points.

- $u_{\mathrm{R}}$ : residual error:

According to (16), the amplitude of $\Delta G D$ varies with $d$, which means the positive parts and negative parts of $\triangle G D$ cannot be counteracted totally when calculating the mean value of $G D_{\mathrm{c} N}$. The residual error is:

$$
\Delta G D_{\mathrm{R}}=\frac{\Delta G D_{1}+\Delta G D_{2}+\cdots+\Delta G D_{N}}{N} .
$$

The expressions of $\Delta G D_{\mathrm{R}}$ and $\Delta G D_{\mathrm{P} 1}$ are calculated according to (16) at $1575.42 \mathrm{MHz}$ and shown in Tab. 4, where $\Delta G D_{\mathrm{P} 1}$ is the first peak amplitude of $\Delta G D$.

According to Fig. 3, $\Delta G D_{\mathrm{P} 1}$ is not more than $0.16 \mathrm{~ns}$, so $\Delta G D_{\mathrm{R}}$ is not more than $0.0011 \mathrm{~ns}$, which is too small to be taken into consideration. $\triangle G D_{\mathrm{R}}$ on other frequency points is similar with this.

- $u_{\mathrm{d}}$ : laser interferometer positioning error:

The laser interferometer positioning error is $\Delta d=0.1 \mu \mathrm{m}$, so the group delay measurement error caused by $\Delta d$ is:

$$
\Delta G D_{\mathrm{d}}=-\frac{\Delta d}{\mathrm{c}}=-3.3 \times 10^{-7} \mathrm{~ns}
$$

which means that the $\Delta G D_{\mathrm{d}}$ is negligible.

\begin{tabular}{|c|c|}
\hline Parameters & Values/Expressions \\
\hline$\left|\Delta G D_{\mathrm{R}}\right|$ & $0.0027 \beta / \mathrm{c}$ \\
\hline$\left|\Delta G D_{\mathrm{P} 1}\right|$ & $0.3957 \beta / \mathrm{c}$ \\
\hline$\left|\Delta G D_{\mathrm{R}}\right| /\left|\Delta G D_{\mathrm{P} 1}\right|$ & 0.0068 \\
\hline
\end{tabular}

Tab. 4. The values and expressions of $\Delta G D_{\mathrm{R}}$ and $\Delta G D_{\mathrm{P} 1}$.

\section{Conclusion}

Accurate antenna GD measurement is becoming more and more important for satellite navigation, remote sensing, modern communication and other military and civil application fields. In this paper, an improved antenna group delay measurement method based on the three antenna extrapolation technique is proposed. A practical group delay measurement for a circularly polarized helical antenna validates the derived multiple-reflection error model, and the detail procedures give an example for implementation of this method. The measurement error due to multiple reflections is reduced by $0.16 \mathrm{~ns}$ compared with the conventional three-antenna method at GPS L1 frequency. The uncertainty of this measurement is evaluated as well, and an expanded uncertainty of $0.20 \mathrm{~ns}$ has been achieved for this case. One more measurement example in frequency band of $(4000 \pm 10) \mathrm{MHz}$ for a standard gain horn antenna is also presented briefly with an expanded uncertainty of $0.12 \mathrm{~ns}$. The implementation of this method for active antenna will be the emphasis of further study.

\section{Acknowledgments}

Authors thank the supports from the National Key Technology Research and Development Program of the Ministry of Science and Technology of China under Grant (2014BAK02B00). They also thank the reviewers for their valuable comments.

\section{References}

[1] IEEE Standard Definitions of Terms for Antennas. IEEE Std 1451993. ISBN: 1-55937-317-2

[2] SONG, Z. F., GENTLE, D., LIN, H. Y., et al. Accurate gain calibration for a WR10 standard gain horn (SGH) using the threeantenna extrapolation technique. In Proceedings of IET International Radar Conference 2015. Hangzhou (China), 2015, p. 1474-1479. DOI: $10.1049 / \mathrm{cp} .2015 .1270$

[3] HAZDRA, P., CAPEK, M., MASEK, M., et al. An introduction to the source concept for antennas. Radioengineering, 2016, vol. 25, no. 1 , p. 12-17. DOI: $10.13164 /$ re.2016.0012

[4] JOHANNSEN, K. G. Measurement of satellite communication parameters: satellite antenna measurements. IEEE Transactions on Instrumentation and Measurement, 1988, vol. 37, no. 2, p. 285 to 291. DOI: $10.1109 / 19.6068$

[5] LEMoine, C., AMAdOR, E., BeSnier, P., et al. Antenna directivity measurement in reverberation chamber from Rician $K$ factor estimation. IEEE Transactions on Antennas and 
Propagation, 2013, vol. 61, no. 10, p. 5307-5310. DOI: 10.1109/TAP.2013.2272691

[6] KIM, J. H., CHOI, H. K. Antenna radiation pattern measurement at a reduced distance. IEEE Transactions on Instrumentation and Measurement, 2005, vol. 54, no. 2, p. 673-675. DOI: 10.1109/CPEM.2004.305447

[7] CERNY, O., DOLECEK, R., KOPECKY, P., et al. Optimization of far-field antenna range. Radioengineering, 2015, vol. 24, no. 4, p. 892-897. DOI: $10.13164 /$ re.2015.0892

[8] MANOHAR, M., KSHETRIMAYUM, R. S., GOGOI, A. K. A compact dual band-notched circular ring printed monopole antenna for super-wideband applications. Radioengineering, 2017, vol. 26, no. 1, p. 64-70. DOI: 10.13164/re.2017.0064

[9] ALI, W., HAMAD, E., BASSIUNY, M., et al. Complementary split ring resonator based triple band microstrip antenna for WLAN/WiMAX applications. Radioengineering, 2017, vol. 26, no. 1 , p. $78-84$. DOI: 10.13164/re.2017.0078

[10] FAKHARIAN, M. M., REZAEI, P., OROUJI, A. A. Polarization and radiation pattern reconfigurability of a planar monopole-fed loop antenna for GPS application. Radioengineering, 2016, vol. 25 , no. 4, p. $680-686$. DOI: $10.13164 /$ re.2016.0680

[11] YU, S., ZHAN, Y. F., PEI, Y. K. The effect of group-delay difference for wideband antenna arraying: degradation and its solution. In Proceedings of 2014 4th IEEE International Conference on Information Science and Technology. Shenzhen (China), 2014, p. 498-501. DOI: 10.1109/ICIST.2014.6920525

[12] RAGHUVANSHI, A., GRAAS, F. V. Impact of antenna group delay variations on protection levels. In Proceedings of 2016 IEEE/ION Position, Location and Navigation Symposium (PLANS). Savannah (USA), 2016, p. 857-862. DOI: 10.1109/PLANS.2016.7479781

[13] LI, L. X., SU, Y. X., LI, B. Y., et al. Phase and group delay analysis for patch antenna. In Proceedings of 2015 Asia-Pacific Microwave Conference (APMC). Nanjing (China), 2015, p. 1-3. DOI: 10.1109/APMC.2015.7413399

[14] LI, L. X., LI, B. Y., CHEN, H. M., et al. GNSS antenna phase center and group delay evaluating. In Proceedings of 2015 IEEE 4th Asia-Pacific Conference on Antennas and Propagation (APCAP). Bali Island (Indonesia), 2015, p. 338-339. DOI: 10.1109/APCAP.2015.7374396

[15] KWON, D. H. Effect of antenna gain and group delay variations on pulse-preserving capabilities of ultrawideband antennas. IEEE Transactions on Antennas and Propagation, 2006, vol. 54, no. 8, p. 2208-2215. DOI: 10.1109/TAP.2006.879189

[16] Minimum Operational Performance Standards (MOPS) for Global Navigation Satellite System (GNSS) Airborne Active Antenna Equipment for the L1 Frequency Band. RTCA DO-301, December $13,2006$.

[17] SHAKHTOUR, H., CORNELIUS, R., HEBERLING, D. Three antenna gain determination method in compact antenna test ranges. In Proceedings of 2013 Loughborough Antennas \& Propagation Conference. Loughborough (UK), 2013, p. 392-396. DOI: 10.1109/LAPC.2013.6711927
[18] FUJII, K., ALEXANDER, M., SUGIURA, A. Uncertainty analysis for three antenna method and standard antenna method. In Proceedings of 2012 IEEE International Symposium on Electromagnetic Compatibility. Pittsburgh (USA), 2012, p. 702-707. DOI: 10.1109/ISEMC.2012.6351648

[19] MILLER, P. The measurement of antenna group delay. In Proceedings of the 8th European Conference on Antennas and Propagation. The Hague (The Netherlands), 2014, p. 1488-1492. DOI: 10.1109/EuCAP.2014.6902064

[20] NEWELL, A. C., BAIRD, R. C., WACKER, P. F. Accurate measurement of antenna gain and polarization at reduced distances by an extrapolation technique. IEEE Transactions on Antennas and Propagation, 1973, vol. 21, no. 4, p. 418-431. DOI: 10.1109/TAP.1973.1140519

[21] Uncertainty of Measurement - Part 3: Guide to the Expression of Uncertainty in Measurement (GUM: 1995). ISO/IEC GUIDE 983: 2008 Ed. 1 .

\section{About the Authors ...}

Haoyu LIN was born in Hebei Province, China. He received his B.Sc. degree in Information and Electronics from Beijing Inst. of Technology, Beijing, China, in 2009 and M.Sc. degree in Astronautics from Beihang University, Beijing, China, in 2012. His research interests include antenna measurement, EMC and WSN.

Zhenfei SONG was born in Jiangsu Province, China. He received his B.Sc. degree in Applied Physics from Shandong University, China, in 2006 and Ph.D. degree in Electromagnetic Compatibility (EM) from Beihang University, Beijing, China in 2012. His current research focuses on precision antenna and microwave field measurement.

Xuetian WANG was born in Jiangsu Province, China. He received the B.Sc. and Ph.D. degrees in Electronic Engineering from Beijing Inst. of Technology, Beijing, China, in 1986 and 2002, respectively. He is currently a full Professor with the School of Information and Electronics, Beijing Inst. of Technology. His current research interests include antenna theory and applications, millimeter-wave imaging, EMC, and terahertz radar.

Hongmin GAO was born in Hebei Province, China. He received the B.Sc. degree in Automation from Hebei Inst. of Technology, Hebei, China in 1992 and Ph.D. degree in Automatic Control from Beijing Inst. of Technology, Beijing, China, in 2002. He is currently a full Professor with the School of Information and Electronics, Beijing Inst. of Technology. His current research interests include millimeter wave THz technology and system. 